\title{
CO-CHANNEL DEPLOYMENT CROSS LAYER APPROACH FOR LTE HETEROGENEOUS NETWORKS
}

\author{
Deepthi Shree A.M ${ }^{1}$, Arun Biradar ${ }^{2}$ \\ ${ }^{1}$ Student, Department of CSE, East West Institute of Technology, Bangalore, Karnataka, India \\ ${ }^{2}$ Head of the Department, Department of CSE, East West Institute of Technology, Bangalore, Karnataka, India
}

\begin{abstract}
Nowadays Heterogeneous Networks (HetNets) are envisioned as the major capacity and performance enhancement enablers by means of increasing the spectral efficiency per unit area. In LTE, since pico cells typically share the frequency band as macro cells, the performance of a low-power pico access node could be severely impaired by interference from a high power macro access node. In co-channel deployment it is necessary to balance the load and Share the resources between the different layers, where macro and pico cells share the same carrier frequency. In this case, one of the main problem that need to be addressed in order to improve the network performance is: Cell selection. For this reason we propose a throughput-based cell selection algorithm. This algorithm targets to optimize the throughput of each user.
\end{abstract}

Keywords: LTE, Heterogeneous Networks, Macro cell, Pico cell and Co-Channel Deployment.

\section{INTRODUCTION}

In recent years, the development of wireless communications has experienced a huge revolution. While just a couple of decades ago mobile phones were mostly used for making calls and sending messages via SMS, the introduction of $3 \mathrm{G}$, which allowed the use of broadband data granting access to browse the internet, and later $4 \mathrm{G}$, which gets higher speeds, marked the development of mobile broadband and data oriented devices such as smartphones and USB modems. These devices are responsible for a fast growth in mobile data traffic.

Mobile networks have to deal with the increase of the number of devices and also the demands of users for higher speeds to allow diversions like real time video streaming and online games. Therefore, mobile networks have to evolve in order to fulfill these demands. In the past years the required increase in capacity would have been achieved by adding more macro nodes in the network. However, the high costs and the space needed for such an approach represent an important problem for the operators. Moreover, spectral efficiency per link is reaching theoretical limits. At the sight of this, LTE-Advanced (LTE-A) in conjunction with Heterogeneous Networks (HetNets) are seen as the keys for further enhance the performance in mobile networks by improving spectral efficiency per unit area.

LTE-A is a technology that was designed to meet the demands of the growing traffic. It includes several advanced techniques such as carrier aggregation, multiple-input multiple-output (MIMO), and the introduction of HetNets. A traditional homogeneous cellular network consists of a group of high-power nodes (macro nodes). Heterogeneous networks instead include not only macro but also low-power nodes. This deployment allows to improve the spectral efficiency per unit area, as the low-power cells make possible to remove coverage holes in the macro-only network and increase the capacity in zones with very high traffic volume. The main characteristic of HetNets is the great disparity between the transmit power used by the highpower and the low-power nodes. This makes necessary the usage of interference management techniques as low-power nodes can suffer great interference from macro ones. A throughtput-based cell selection algorithm is proposed to optimize the throughput of each user.This is achieved by choosing the best cell according to the estimated user throughput that takes into account both the number of users and the signal quality of the candidate cell, that means received signal strength quality.



Fig -1 Architecture of LTE heterogeneous Networks

\section{PROBLEM STATEMENT}

In a heterogeneous network(Hetnet) a series of low power pico nodes are distributed through the existing macro cell network. The performance of low power pico nodes could be severely impaired by interference from high power macro nodes. In these networks when a new user equipment(UE) enters in the networks determining the association rules that decide which UEs should associated with which cells should 
be considered in efficient way. In this way a throughputbased cell selection approach is a one of the good way to improve the network performance.

\section{RELATED RESEARCH WORK}

In a co-channel deployment both macro and small cells share the same frequency carrier. This may be the only option if the available spectrum is limited. One of the key feature to consider for the design of the network is UE cell selection. There are few related works has been performed on UE cell selection.

\subsection{UE Cell Selection}

In traditional homogeneous networks, the way of selecting cell for an arriving UE is based on the UE measurement of the received signal strength. The serving cell selected is the one with the highest signal. There are different signal strength indicators.

- Reference Signal Received Power (RSRP): which measures for each cell the average of the power of the resource elements that carry cell-specific reference signals within the considered measurement frequency bandwidth. This indicator reflects the received power from each cell.

- Reference Signal Received Quality (RSRQ): which is calculated dividing RSRP by the total received power, thus including the channel quality of the resources. This indicator provides additional information useful in cases when RSRP is not reliable enough for doing the cell selection or a handover.

Usually in co-channel deployments the cell selection is done based on the RSRP level. However, it is worth noticing that macro cells have much larger coverage areas than the small cells due to the large transmit power disparity between the macro and small cells, resulting in few users served by the small cells. It is desirable for the network to balance the load between the macro and pico layers to harvest the gain from cell splitting.

\subsection{Load Based Cell Selection}

The load-based cell selection approach focus on the load in each cell as decision criterion.

Advantages: It considers the number of users divided by the capacity of each layer as load measurement.

Disadvantages: This approach has the disadvantage of not taking into account the channel quality in each cell.

\subsection{Cell Selection Technique using RE}

The cell selection technique using $\mathrm{RE}$ (Range expansion) concentrate in the SINR(signal interference plus noise ratio), in which the cell is selected with highest SINR, measuring it during normal transmission subframes for macro cells and during ABS subframes for pico cells.

Disadvantage: In this case the load in each cell is not considered.

\section{PROPOSED SYSTEM}

\subsection{System Architecture}

In propose system, throughput-based cell selection approach is introduced. Throughput-based cell selection algorithms aim to balance the load among the cells by selecting the one which offers best throughput, thus taking into account both the load in each cell and the channel quality. The throughput in each cell can be estimated based on a function of SINR and number of users. This algorithm depends strongly on the current condition of the network when choosing the serving cell. Fig. 2 shows the architecture of our proposed idea.

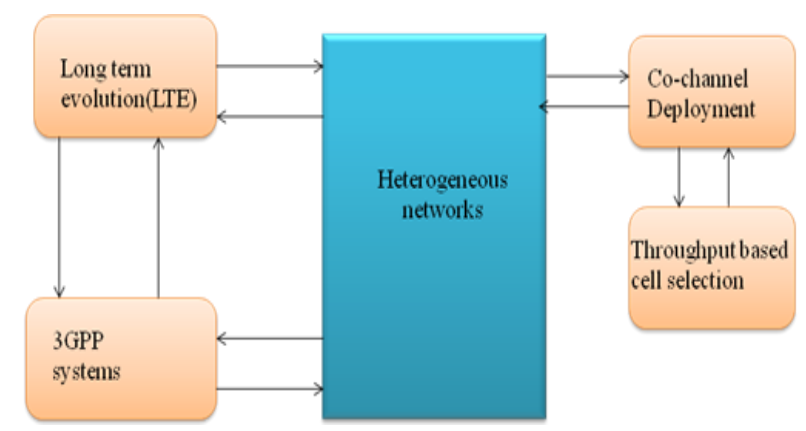

Fig -2: System architecture of proposed approach.

\subsection{Data Flow Diagram}

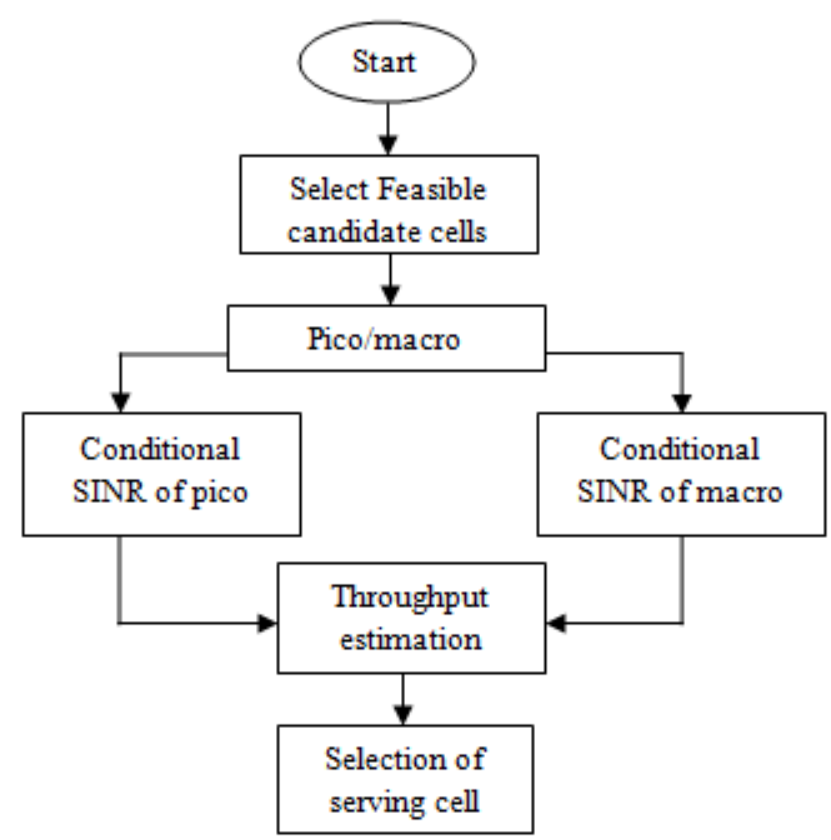

Fig -3: Data flow diagram of throughput based algorithm.

\section{IMPLEMENTATION}

The throughput-based algorithm targets to optimize the throughput of each user. This is achieved by choosing the best cell according to the estimated user throughput that takes into account both the number of users and the signal quality of the candidate cell. This algorithm assumes that the $\mathrm{PF}$ scheduler is applied, thus sharing the resources fairly between all the users connected to a certain cell. 


\subsection{Set of Feasible Candidate cells F Composition}

The set of feasible candidate cells, F, is composed by every cell $\mathrm{i}$ which fulfills the following condition:

\section{$($ PRxmax / PRxi $)<\left(10^{\wedge}(\right.$ RETHRESHOLD /10) $)$}

Where:

- PRxi is the power received by the UE from cell $\mathrm{i}$.

- PRxmax is the maximum power received by the UE, corresponding to the strongest signal the UE receives i.e. $\operatorname{maxPRxi}$.

- RETHRESHOLD $(\mathrm{dB})$ is the maximum threshold allowed between the maximum power received by the UE and $P R x i$.

The parameter RETHRESHOLD sets a condition check for the selection of the candidate base stations. The objective of this parameter is preventing the connection of the UE to a base station(BS) with a very bad signal quality. This could happen in a case where the network is very loaded and then a BS with poor signal but with few users shows the best theoretical throughput. In practice, connecting to a BS with poor signal causes communication problems, and should be avoided. The RETHRESHOLD value used in this study for the throughput-based cell selection algorithm is $15 \mathrm{~dB}$.

The conditional SINR is stable, and this stability is important for the good performance of the throughput-based algorithm. However, the conditional SINR is something ideal that cannot be obtained in the real network. Each UE can measure the channel quality in a certain moment. At that moment, some BSs are transmitting and others are not. In some other moment, the BSs transmitting may be different. Thus, it is not possible for the UE to determine if all BSs are transmitting at full power in order to obtain the conditional SINR. It would be interesting to study in the future how to adapt this algorithm so that it can be applied to a real network.

\subsection{Conditional SINR Estimation}

The conditional Signal to interference plus noise ratio (SINR) is estimated for each macro and pico cells separately. The conditional SINR for macro cells are computed as follows:

$$
\Gamma i=\text { PRxi / (Interference }+ \text { Noise })
$$

The conditional SINR for pico cells are computed as follows:

$$
\Gamma \mathrm{i}=\text { PRxi / (Interference }- \text { PRxstrongest_macro + Noise })
$$

Where:

- PRxMacroi is the power received by the UE from Macro "i".

- PRxPicoi is the power received by the UE from Pico " $\mathrm{i}$ ".

- PRxStrongest Macro is the power received by the UE corresponding to the strongest macro signal. This is the strongest interferer for the potential pico UE.

- Interference is the total interference the UE suffers assuming all BSs active.
- Noise is the thermal noise.

$\Gamma i$ estimates the SINR the UE would have by connecting to a certain base station with the assumption that the rest of base stations are transmitting at full power (that is, considering all BSs to be active). In the case of pico cells the conditional SINR is computed assuming that the dominant macro interferer is muted i.e. uses ABS all the time. Thus, this estimation is optimistic for the pico cells as it is subtracted the power of the strongest macro interferer. Besides, the estimation is also optimistic for the macro base stations as it is not considered that in muted subframes there is no transmission available from the macro.

This approach for the estimation of the throughput does not take into account the applied muting ratio in each cell. Therefore, the cell selection is based just on the number of users and the signal quality, which allows the algorithm to balance the load among the cells, leaving the Fast ABS to manage the resource partitioning.

\subsection{Estimated Throughput Calculation}

The estimated throughput the UE would get by connecting to a certain candidate base station " $\mathrm{i}$ " is calculated according to the following formula:

$$
\mathrm{Ri}=(1 /(\mathrm{Ni}+1)) * \mathrm{BWi} * \log 2(1+\Gamma \mathrm{i})
$$

Where:

- $N i$ is the number of users in cells $i$.

- $B W i$ is the component carrier bandwidth of cell $\mathrm{i}$.

- $\Gamma i$ is the conditional SINR of the UE in cell $\mathrm{i}$.

\subsection{Serving Cell Selection}

Every time when an UE arrives to the network, the serving cell $i^{*}$ is selected as follows:

$$
i^{*}=\underset{i \in F}{\arg \max }\left\{\hat{R}_{i}\right\}
$$

Where:

- $R i$ is the estimated throughput of the UE in cells $i$.

- $F$ is the set of feasible candidate cells for the UE.

\subsection{Pseudo-Code for Throughput Based Cell Selection Algorithm}

The pseudo-code for throughput-based cell selection algorithm is represented as follows:

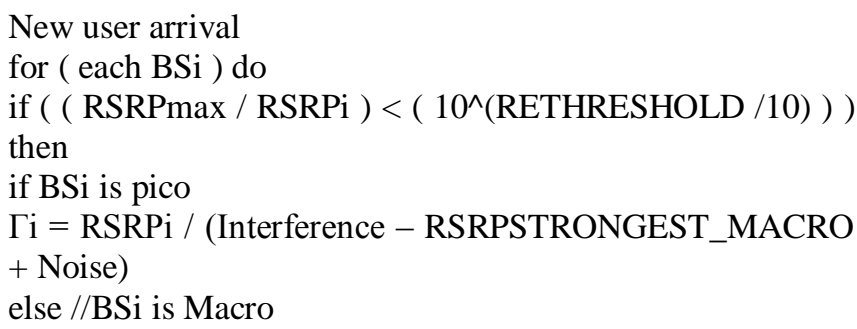


$\Gamma \mathrm{i}=\mathrm{RSRPi} /$ (Interference + Noise)

end if;

$\mathrm{Ri}=(1 /(\mathrm{Ni}+1)) * \mathrm{BWi} * \log 2(1+\Gamma \mathrm{i})$

end if;

end for;

Select BS with $\max \{\mathrm{Ri}\}$

Where:

- BSi: every BS within reach of the new UE.

- RSRPi: RSRP that UE receives from BSi.

- RSRPmax: best RSRP, corresponding to the strongest signal the UE receives.

- RSRPSTRONGEST_MACRO: RSRP that UE receives from the macro cell with strongest signal.

- RETHRESHOLD (dB): maximum difference allowed between best RSRP and RSRPi.

- Interference: total interference the UE suffers assuming all base stations transmitting at full power.

- Noise: thermal Noise.

- Гi: Geometry Factor the UE would obtain connecting to BSi.

- Ni : number of users connected to BSi.

- BWi: Component Carrier Bandwidth of BSi.

- Ri: estimated throughput the UE would obtain connecting to BSi.

\subsection{Advantages}

In Heterogeneous Network, the Throughput-Based cell selection approach determines the association rules that decides which UEs should associate with which picos. This approach mitigates the interference problem in an efficient way. It does not considers the distance between nodes, it performs scheduling based on the user equipment throughput in each candidate cells. Thus it increases the overall performance of the network.

\section{CONCLUSION}

LTE is the viable path for mobile broadband. In LTE heterogeneous networks the network performance can be improved by using throughput-based algorithm especially in the case of co-channel deployment. This dynamic algorithm gives a good solution for UE association rules where each new entering UE can be associated with a cell based on its throughput.

\section{REFERENCES}

[1] Magazine, pp. 10-21, June 2011.J. Clerk Maxwell, A Treatise on Electricity and Magnetism, 3rd ed., vol. 2. Oxford: Clarendon, 1892, pp.68-73. A. Damnjanovic, J. Montojo, Y. Wei, T. Ji, T. Luo, M. Vajapeyam, T. Yoo, O. Song and D. Malladi, "A Survey on 3GPP systems".

[2] K. I. Pedersen, Y. Wang, S. Strzyz and F. Frederiksen, "Enhanced Inter Cell Interference Coordination in Co-Channel Multi-Layer LTE Advanced Networks," IEEE Wireless Communications Magazine, pp.120-127, June 2013.
[3] S. Brueck, "Heterogeneous Networks in LTEAdvanced," in 2011 8th Symposium on Wireless Communication Systems, Aachen, 2011.

[4] Q. Ye, B. Rong, Y. Chen, C. Caramanis and J. G. Andrews, "Towards and Optimal User Association in Heterogeneous Cellular Networks," in IEEE Global Communications Conference (GLOBECOM), 2012.

[5] D. López-Pérez, I. Güvenç, G. De la Roche, M. Kountouris, T. Q. Quek and J. Zhang, "Enhanced Intercell Interference Coordination Challenges in Heterogeneous Networks," IEEE Wireless Communications Magazine, pp. 22-30, June 2011.

[6] B. Soret, H. Wang, K. I. Pedersen and C. Rosa, "Multicell Cooperation for LTE-Advanced Heterogeneous Network Scenarios," IEEE Wireless Communications Magazine, February 2013.

\section{BIOGRAPHIES}

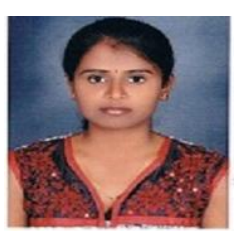

Deepthi Shree A.M received her B.E in computer science from Govt. engineering college hassan, and pursuing her M.Tech in CS in EWIT Bangalore.

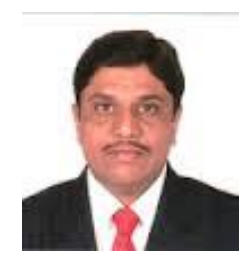

Dr. Arun Biradar is HOD of the computer science department in EWIT bangalore. His area of specializations are computer networks, wireless networks. 